\title{
Thermal fission rate around super-normal phase transition
}

\author{
K. Hagino, ${ }^{1}$ N. Takigawa, ${ }^{1}$ and $M \cdot$ Abe $^{2}$ \\ 1 Department of Physics, Tohoku University, 980-77 Sendai, Japan \\ 2 Faculty of Science and Engineering, \\ Ishinomaki Senshu University, 986 Ishinomaki, Japan
}

\begin{abstract}
Using Langer's $I m F$ method, we discuss the temperature dependence of nuclear fission width in the presence of dissipative environments. We introduce a low cut-off frequency to the spectral density of the environmental oscillators in order to mimic the pairing gap. It is shown that the decay width rapidly decreases at the critical temperature, where the phase transition from super to normal fluids takes place. Relation to the recently observed threshold for the dissipative fission is discussed.

PACS number(s): 24.75.+i, 25.70.Jj, 24.60.Dr, 05.40.+j
\end{abstract}


Fission of a hot nucleus has attracted much interests of nuclear physicists in the past several years to study nuclear dissipation together with deeply inelastic heavy-ion scattering[1-3]. It is known that statistical codes to calculate the dacay of a compond nucleus significantly underestimate the experimentally observed prefission neutron, charged particle, and $\gamma$-ray multiplicities at high excitation energies if the original Bohr-Wheeler formula for the fission width is used, though it works pretty well at low energies[4-7]. One way to understand this fact is to consider that fission is hinderd by nuclear dissipation. Based on this idea, Thoennessen and Bertsch have analysed fission data on prefission neutron, charged particle and $\gamma$ ray multipicities for various systems by using statistical codes, and obtained systematics of the threshold for dissipative fission 4 , 5. This systematics has been confirmed by experimentally studying the excitation energy dependence of the fission probability in ${ }^{200} \mathrm{~Pb}$ compound nuclei[6].

On the other hand, the nuclear dissipation does not play any significant role in spontaneous fission because of the strong pairing correlation between nucleons 8, 9]. When one discusses nuclear fission at moderate excitation energies, one has to take into account the temperature dependence of the pairing gap. The pairing gap decreases with temperature and the nucleus eventually undergoes a phase transition from a superfluid to a normal fluids[10-13]. It is the purpose of this paper to investigate the effects of the super-normal phase transition on the fission width at finite temperatures. To this end, we use Langer's $I m F$ method, where the decay width of a metastable state is related to the imaginary part of the free energy[14-18]. In this method one can describe the decay process for a very wide range of temperature, i.e. from zero temperature, where the decay process is governed by the quantum tunneling, to high temperatures, where thermal decay dominates [19]. Also, the method can be applied to a system with many degrees of freedom 15. 
We consider a system where a macroscopic degree of freedom $q$ is coupled to environmental heat bath. In the problem of fission, $q$ corresponds to the fission coordinte. We assume the following Lagrangian for this system [20]

$$
L=\frac{1}{2} M(q) \dot{q}^{2}-V(q)+\sum_{i} \frac{1}{2} m_{i}\left(\dot{x}_{i}^{2}-\omega_{i}^{2} x_{i}^{2}\right)-\sum_{i} c_{i} x_{i} f(q)+\sum_{i} \frac{c_{i}^{2} f(q)^{2}}{2 m_{i} \omega_{i}^{2}}
$$

where $\left\{x_{i}\right\}$ and $\left\{\omega_{i}\right\}$ are the coordinates of the environmental oscillators and the corresponding excitation energies, respectively. $V(q)$ is a potential for the macroscopic degree of freedom, which has a local minimum and a maximum at $q=q_{0}$ and $q=q_{b}$, respectively. $M(q)$ and $f(q)$ are the mass of the macroscopic motion and the coupling form factor, respectively. We assume general functions of $q$ for them [16, 18]. The last term is the so called counter term which cancels the static potential renormalization due to the coupling between the macroscopic and the environmental degrees of freedom [20]. Takigawa and Abe have suggested that, in contrast to heavy-ion fusion reactions at subbarrier energies where the static potential renormalization plays an important role in enhancing the fusion cross section over the predictions of a one dimensional potential model[21, 22], the static potential renormalization in the fission problem can lead to two opposite effects, i.e. it could either lower or increase the effective fission barrier compared with the bare potential barrier, thus leading to either hindrance or enhancement of the fission rate, depending on the properties of the coupling form factor $f(q)$ [16]. Both cases lead to a temperature dependent fission barrier hight [1, [5]. In this paper, however, we introduce the counter term because we do not know yet much about the coupling form factor.

In order to obtain the free energy, we first express the partition function in the path integral form. After integrating out the environmental degrees of freedom, the partition function at the temperature $k_{B} T=1 / \beta$ takes the form [23]

$$
Z(\beta)=\int \mathcal{D}[q(\tau)] e^{-S_{e f f}[q(\tau)] / \hbar},
$$


where the path integral is performed over all the periodic paths with the period $\beta \hbar$. The effective Euclidean action $S_{\text {eff }}[q(\tau)]$ is given by

$S_{e f f}[q(\tau)]=\int_{0}^{\beta \hbar} d \tau\left(\frac{1}{2} M(q(\tau)) \dot{q}^{2}+V(q(\tau))\right)+\frac{1}{2} \int_{0}^{\beta \hbar} d \tau \int_{0}^{\beta \hbar} d \tau^{\prime} k\left(\tau-\tau^{\prime}\right) f(q(\tau)) f\left(q\left(\tau^{\prime}\right)\right)$

with the influence kernel $k(\tau)$ [16, 23]

$$
k(\tau)=\sum_{i}\left[\frac{c_{i}^{2}}{m_{i} \omega_{i}^{2}}: \delta(\tau):-\frac{c_{i}^{2}}{2 m_{i} \omega_{i}} \frac{\cosh \left[\omega_{i}\left(|\tau|-\frac{1}{2} \beta \hbar\right)\right]}{\sinh \left(\frac{1}{2} \hbar \omega_{i} \beta\right)}\right]
$$

where

$$
: \delta(\tau):=\sum_{n=-\infty}^{\infty} \delta(\tau-n \beta \hbar)
$$

is a generalized delta function with period $\beta \hbar$.

We consider now a high temperature regime, where the decay of a metastable state is governed by the thermal hopping. Evaluating the path integral in eq.(2) in the saddle point approximation and using the relation between the decay width $\Gamma$ and the imaginary part of the free energy 14

$$
\Gamma(T)=-\frac{2}{\hbar} \frac{T_{c}}{T} \operatorname{Im} F
$$

$T_{c}$ being the cross over temperature where the transition between the thermal activated decay and the quantum tunneling occurs, we find that the decay width at temperature $T$ can be expressed as 16

$$
\Gamma=\frac{\omega_{0}}{2 \pi} \frac{\omega_{R}}{\omega_{b}} \sqrt{\frac{M\left(q_{0}\right)}{M\left(q_{b}\right)}} f_{q} e^{-\beta V_{b}},
$$

where $\omega_{R}$ is defined as $2 \pi k_{B} T_{c} / \hbar . \omega_{0}, \omega_{b}$, and $V_{b}$ are the curveture of the potential barrier $V(q)$ at the local minimum $q=q_{0}$, that at the barrier position $q_{b}$, and the height of the potential barrier i.e., $V_{b}=V\left(q_{b}\right)-V\left(q_{0}\right)$, respectively. $f_{q}$ is the quantum correction factor due to the quantum fluctuation of the paths around the classical paths $q_{c \ell}(\tau)=q_{b}$, 
$q_{c \ell}(\tau)=q_{0}$, and is given by

$$
f_{q}=\prod_{n=1}^{\infty} \frac{\nu_{n}^{2}+\omega_{0}^{2}+\left(\frac{d f}{d q}\right)_{q=q_{0}}^{2} \nu_{n} \hat{\gamma}_{0}\left(\nu_{n}\right)}{\nu_{n}^{2}-\omega_{b}^{2}+\left(\frac{d f}{d q}\right)_{q=q_{b}}^{2} \nu_{n} \hat{\gamma}_{b}\left(\nu_{n}\right)}
$$

where $\nu_{n}=2 \pi n / \beta \hbar$ is the Matsubara frequencies. $\hat{\gamma}$ is the Laplace transform of the retarded friction kernel [16], and is given by

$$
\hat{\gamma}(z)=\frac{1}{M(q)} \sum_{i} \frac{c_{i}^{2}}{m_{i} \omega_{i}^{2}} \frac{z}{z^{2}+\omega_{i}^{2}}
$$

The subscripts 0 and $b$ in eq.(8) denote that the quantities with those indices should be evaluated at $q=q_{0}$ and $q=q_{b}$, respectively. The crossover temperature $T_{c}$ is identified with the highest temperature at which the quantum correction factor $f_{q}$ diverges 15. In the absence of environments, this prescription assigns $k_{B} T_{c}$ to be $\hbar \omega_{b} / 2 \pi$. This is consistent with the earlier observation by Affleck on the crossover temperature [14]. It should be noticed that Langer's ImF method implicitly assumes that the coupling of the macroscopic degree of freedom to the environmental degrees of freedom is strong enough to assure that the system is always in a thermal equillibrium.

We now apply eq. (7) to the problem of the fission of a hot nucleus. Following ref. 8 we introduce a low cutoff frequency $\omega_{c}$ to the distribution of the environmental oscillators in order to mimic that there is no nuclear levels below the two quasi particle state in even-even nuclei. Accordingly, we set the cutoff frequency to $2 \Delta(T) / \hbar, \Delta(T)$ being the pairing gap at the temperature $T$, and take the spectrum density of the environmental oscillators as 8

$$
J(\omega) \equiv \frac{\pi}{2} \sum_{i} \frac{c_{i}^{2}}{m_{i} \omega_{i}} \delta\left(\omega-\omega_{i}\right)=\eta\left(\omega-\omega_{c}\right) \theta\left(\omega-\omega_{c}\right)
$$

where $\eta$ is the friction constant [20]. Note that $\omega_{c}=\infty$ and $\omega_{c}=0$ correspond to two extreme cases where there is no dissipation at all and where the spectrum density is given by the usual Ohmic dissipation, respectively. The former and the latter cases 
give the Bohr-Wheeler formula and the well known Kramers's formula at moderate to strong friction for the decay rate, respectively, with a quantum correction factor 15. The temperature dependence of the cutoff frequency $\omega_{c}$ is given by solving the thermal gap equation[10-13]

$$
\Delta=\frac{G}{2} \sum_{k} \frac{\Delta}{\sqrt{\left(\epsilon_{k}-\lambda\right)^{2}+\Delta^{2}}} \tanh \left(\frac{\sqrt{\left(\epsilon_{k}-\lambda\right)^{2}+\Delta^{2}}}{2 k_{B} T}\right)
$$

where $G$ and $\lambda$ is the strength of the pairing interaction and the chemical potential, respectively. For the spectrum density given by eq.(10), eq.(9) for the Laplace transform of the damping kernel reads

$$
\hat{\gamma}(z)=\frac{\eta}{M(q)}+\frac{2}{\pi} \frac{\eta}{M(q)}\left(\frac{\omega_{c}}{z} \log \frac{\omega_{c}}{\sqrt{\omega_{c}^{2}+z^{2}}}-\tan ^{-1} \frac{\omega_{c}}{z}\right)
$$

Note that the second term in this equation vanishes when the cutoff frequency $\omega_{c}$ is set zero.

We now apply the above arguments to the fission of ${ }^{248} \mathrm{Cf}$. We take the reduced mass for the symmetric fission for $M(q)$ and the potential given in ref. 24] for $V(q)$. $\hbar \omega_{0}, \hbar \omega_{b}, q_{b}$ and $V_{b}$ then take the values of $1.18 \mathrm{MeV}, 1.06 \mathrm{MeV}, 3.4 \mathrm{fm}$, and $3.67 \mathrm{MeV}$, respectively. We take the value $20 \times 10^{21} /$ sec. [1] for the reduced dissipation coefficient $\beta \equiv \eta / M$, and assume a bi-linear coupling form factor i.e., $f(q)=q$. Since we are interested in the effects of pairing in the super to normal transition region, we use a simplified expression for the temperatre dependent pairing gap,

$$
\begin{aligned}
\Delta(T) & =k_{B} T_{c}^{\text {pair }} \sqrt{\frac{8 \pi^{2}}{7 \zeta(3)}\left(1-\left(T / T_{c}^{\text {pair }}\right)\right.} \quad\left(\text { for } T<T_{c}^{\text {pair }}\right) \\
& =0 \quad\left(\text { for } T>T_{c}^{\text {pair }}\right)
\end{aligned}
$$

which is valid near the transition temperature 13. In eq.(13) $\zeta$ is the zeta function and $T_{c}^{\text {pair }}$ the critical temperature for the super-normal phase transition. We assign the pairing 
gap at zero temperature to be $12 / \sqrt{A}, A$ being the mass number of a nucleus, and estimate the critical temperature $T_{c}^{\text {pair }}$ using the relation $T_{c}^{\text {pair }} \sim 0.567 \Delta_{0}[12,13]$.

Figure 1 shows the crossover temperature $T_{c}$ as a function of the cutoff parameter $\hbar \omega_{c}$. This is given by solving

$$
\omega_{R}^{2}+\omega_{R} \frac{\eta}{M}-\omega_{b}^{2}+\frac{\eta}{M}+\frac{2}{\pi} \frac{\eta}{M}\left(\frac{\omega_{c}}{\omega_{R}} \log \frac{\omega_{c}}{\sqrt{\omega_{c}^{2}+\omega_{R}^{2}}}-\tan ^{-1} \frac{\omega_{c}}{\omega_{R}}\right)=0
$$

It should be remarked that in calculating the decay rate based on eq.(7) the crossover temperature $\omega_{R}$ has to be evaluated at each temperature $T$ with corresponding cuttoff frequency $\omega_{c}$ i.e., one must solve eq.(15) by treating $\omega_{c}$ as though it is independent of temperature. Otherwise, one cannot recover the decay rate formula of Kramers modified by the quantum correction factor at temperatures higher than $T_{c}^{\text {pair }}$, where the pairing gap vanishes. The solid line in fig. 1 is the solution of eq.(15). The dashed line is the crossover temperature in the absence of environments, i.e. $\hbar \omega_{b} / 2 \pi$. If one sets $\omega_{c}$ to be zero, the crossover temperature is given by $\left(\sqrt{1+\alpha^{2}}-\alpha\right) \hbar \omega_{b} / 2 \pi$, $\alpha$ being $\eta / 2 M \omega_{b}$ [15]. This value is denoted by the dotted line in the figure. The crossover temperature gradually decreases as the cut-off frequency decreases reflecting the increasing dissipation 8.

Figure 2 shows the quantum correction factor given by eq.(8) as a function of the temperature. In the limits of $\omega_{c} \rightarrow 0$ and $\infty$, the infinite product in eq.(8) can be simplified by using $\Gamma$ function [15, 17]. In the case of finite $\omega_{c}$, one has to evaluate it directly until one gets convergence. In general cases, however, this is a fairly difficult numerical task because the ratio for each $n$ in eq.(8) never becomes sufficiently close to one even for very large $n$. Consequently, numerical errors accumulate as one performs the production many times. In our applications, where we used a constant mass and a bilinear coupling, the infinite product series converged. The dashed and the dotted lines are the quantum correction factor in the limit of $\omega_{c} \rightarrow 0$ and $\infty$, respectively. The solid 
line is the quantum correction factor when the lower cutoff for each temperature has been introduced. The left and the right arrows in the figure show the crossover temperature from a quantal to a thermal decay, i.e. $T_{c}=0.169 \mathrm{MeV}$, in the absence of environment and the transition temperature from super to normal fluids, i.e. $T_{c}^{\text {pair }}=0.432 \mathrm{MeV}$. The solid line coincides with the dotted line at temperatures higher than $T_{c}^{\text {pair }}$, as is expected. Note that the quantum correction factor approaches to one at high temperatures.

The decay rate for this system is shown in figure 3 as a function of the temperature. The meaning of each line is the same as that in fig.2. We observe a sudden decrease of the decay rate at the critical temperaure $T_{c}^{\text {pair }}$. This behaviour agrees with that found in ref. 25], where the diffusion of a heavy particle in metal was studied by taking a superconducting phase transition of the environmental electrons into account. Notice that the cusp behaiour in the transitional region will be smeared out to some extent in actual cases, for example, by the gradual disappearance of the pairing gap with temperature.

In summary, we made use of the $I m F$ method of Langer to discuss the fission dynamics of hot nuclei in the presence of a dissipative environment. We modified the CaldeiraLeggett model by introducing a low cutoff frequency in order to mimic the effects of nuclear superfluidity due to pairing interaction. We took into account the temperature dependence of the pairing gap, and thus the phase transition from a super to a normal liquids. The cutoff makes the dissipation weak. This accords with the fact that the nuclear dissipation plays less or no significant role in nuclear fission at low temperatures [26]. The pairing gap gets smaller as the temperature increases. We suggested that the decay rate suddenly decreases at the critical temperature, where the pairing gap disappears. This could be related to the sudden decay of superdeformed band at some critical angular momentum [27.

In this paper, we assumed the standard value for the pairing gap parameter. The 
critical temperature was then found to be much lower than the threshold temperature for the dissipative fission discussed in ref. [4]. The non-monotonic behaviour of the decay rate shown in Fig.3 in this paper might therefore indicate the existence of the second critical temperature other than the threshold temperature discussed in ref. [4]. The time dependent Hartree Fock (TDHF) calculations for the induced fission of ${ }^{236} \mathrm{U}$ with the constraint of axial symmetry uses, however, fairly large values of the effective pairing gap which are about 2.5 to 7.5 times larger than the usual values 28]. The authors in ref.[28] claim that these large values reflect the effect of the breaking of the axial symmetry. If we use such large effective pairing gaps in our calculations, the critical temperature, where the sudden decrease of the fission rate due to the disappearance of the pairing gap occurs, nearly coincides with the threshold temperature found in ref. [G]. In order to draw a definite conclusion to this problem more detailed studies of the coupling form factor and of the temperature and the coordinate dependence of the friction constant are required. The work toward this direction is now in progress.

The authors thank Profs. H.A. Weidenmüller and D.M. Brink for useful discussions. The work of K.H. was supported by Research Fellowships of the Japan Society for the Promotion of Science for Young Scientists. This work was supported by the Grant-inAid for General Scientific Research, Contract No.06640368, and the Grant-in-Aid for Scientific Research on Priority Areas, Contract No.05243102, from the Japanese Ministry of Education, Science and Culture. 


\section{Figure Captions}

Fig.1: The cutoff frequency dependence of the cross over temperature $T_{c}$ between the quantum and the thermal regimes. The solid line was obtained by numerically solving eq.(15). The dashed and the dotted lines are the crossover temperature in the absence of environments and that in the system with Ohmic dissipation without cutoff, respectively. Fig.2: Quantum correction factor as a function of temperature. The dashed and the dotted lines are the quantum correction factor in the absence of environment and that in the system with Ohmic dissipation without cutoff, respectively. The solid line is the quantum correction factor when a lower cutoff frequency has been introduced throuth the temperature dependence of the pairing gap. The left and the right arrows are the crossover temperature from a quantal to a thermal decay, and the critical temperature for the super to normal phase transition, respectively.

Fig.3: Decay rate as a function of temperature. The dashed and the dotted lines are the decay rate in the absence of environment and in the Kramers limit, where there is no cutoff, respectively. The solid line takes the effects of cutoff into account. 


\section{References}

[1] D. Boilley, E. Suraud, Y. Abe, and S. Ayik, Nucl. Phys. A556, 67(1993).

[2] T. Wada, Y. Abe, and N. Carjan, Phys. Rev. Lett. 70, 3538(1993).

[3] D.J. Hinde, D. Hilscher, and H. Rossner, Nucl. Phys. A502, 497c(1989).

[4] M. Thoennessen and G.F. Bertsch, Phys. Rev. Lett. 71, 4303(1993).

[5] R. Vandenbosch, Phys. Rev. C50, 2618(1994).

[6] D. Fabris et al., Phys. Rev. Lett. 73, 2676 (1994).

[7] P. Fröbrich, I.I. Gontchar, and N.D. Mavlitov, Nucl. Phys. A556, 281(1993).

[8] N.R. Dagdeviren and H.A. Weidenmüller, Phys. Lett. B186, 267(1987).

[9] F. Barranco, G.F. Bertsch, R.A. Broglia, and E. Vigezzi, Nucl. Phys. A512, 253(1990).

[10] P. Lotti, F. Cazzola, P.F. Bortignon, R.A. Broglia, and A. Vitturi, Phys. Rev. C40, 1791(1989).

[11] F. Alassia, O. Civitarese, amd M. Reboiro, Phys. Rev. C35, 812(1987).

[12] O. Civitarese, G.G. Dussel, and R.P.J. Perazzo, Nucl. Phys. A404, 15(1983).

[13] A. Iwamoto and W. Greiner, Z. Phys. A292, 301(1979).

[14] J.S. Langer, Ann. Phys. (N.Y.) 41, 108(1967); G. Callan and S. Coleman, Phys. Rev. D16, 1762(1977); I.K. Affleck, Phys. Rev. Lett. 46, 388(1981).

[15] H. Grabert, P. Olschowski and U. Weiss, Phys. Rev. B36, 1931(1987).

[16] N. Takigawa and M. Abe, Phys. Rev. C41, 2451(1990).

[17] P. Fröbrich and G.-R. Tillack, Nucl. Phys. A540, 353(1992).

[18] J.-D. Bao, Y.-Z. Zhuo and X.-Z. Wu, Phys. Lett. B327, 1(1994); Z. Phys. A347, 217(1994). 
[19] P. Hänggi, P. Talkner,and M. Borkovec, Rev. Mod. Phys. 62, 251(1990).

[20] A.O. Caldeira and A.J. Leggett, Phys.Rev.Lett. 46, 211(1981).

[21] N. Takigawa, K. Hagino, M. Abe and A.B. Balantekin, Phys.Rev.C49, 2630(1994).

[22] N. Takigawa, K. Hagino, and M. Abe, Phys. Rev. C51, 187(1995).

[23] H. Grabert, P. Schramm, and G.-L. Ingold, Phys. Rep. 168, 115(1988).

[24] P. Grangé, L. Jun-Qing, and H.A. Weidenmüller, Phys. Rev. C27, 2063(1983).

[25] H. Yoshioka, K. Awaka, and H. Fukuyama, (unpublished).

[26] J.R. Nix, Nucl. Phys. A502, 609c(1989).

[27] Y.R. Shimizu, E. Vigezzi, T. Døssing, amd R.A. Broglia, Nucl. Phys. A557, 99c(1993).

[28] J.W. Negele, S.E. Koonin, P. Möller, J.R. Nix, and A.J. Sierk, Phys. Rev. C17, 1098(1978). 